\title{
BMJ Open Low-value injury care in the adult orthopaedic trauma population: a protocol for a rapid review
}

\author{
Mélanie Bérubé (D) , , 2 Lynne Moore, ${ }^{1,3}$ Stéphane Leduc, ${ }^{4}$ Imen Farhat, ${ }^{1}$ \\ Martin Lesieur, ${ }^{5}$ Jean Lamontagne, ${ }^{5}$ Stéphane Pelet, ${ }^{5}$ Gregory Berry, ${ }^{6}$ \\ Pier-Alexandre Tardif (10 , ${ }^{1}$ Caroline Côté, ${ }^{2}$ Jérôme Paquet ${ }^{7}$
}

To cite: Bérubé M, Moore L, Leduc $\mathrm{S}$, et al. Low-value injury care in the adult orthopaedic trauma population: a protocol for a rapid review. BMJ Open 2020;10:e033453. doi:10.1136/ bmjopen-2019-033453

- Prepublication history and additional material for this paper are available online. To view these files, please visit the journal online (http://dx.doi org/10.1136/bmjopen-2019033453).

Received 05 August 2019 Revised 26 December 2019 Accepted 19 February 2020

Check for updates

(C) Author(s) (or their employer(s)) 2020. Re-use permitted under CC BY-NC. No commercial re-use. See rights and permissions. Published by BMJ.

For numbered affiliations see end of article.

Correspondence to

Professor Mélanie Bérubé;

melanie.berube@fsi.ulaval.ca

\section{ABSTRACT}

Introduction Orthopaedic injuries affect almost $90 \%$ of trauma patients. A previous scoping review and expert consultation survey identified 15 potential low-value intra-hospital practices in the adult orthopaedic trauma population. Limiting the frequency of such practices could reduce adverse events, improve clinical outcomes and free up resources. The aim of this study is to synthesise the evidence on intra-hospital practices for orthopaedic injuries, previously identified as potentially of low value. Methods and analysis We will search Medline, Excerpta Medica Database (EMBASE), the Cochrane Central Register of Controlled Trials and Epistemonikos to identify systematic reviews, randomised controlled trials (RCTs), quasi-RCTs, cohort studies and case-control studies that evaluate selected practices according to a priori PICOS statements (Population-InterventionComparator-Outcome-Study design). We will evaluate the methodological quality for systematic reviews using the Measurement Tool to Assess Systematic Reviews version 2 (AMSTAR-2). Risk of bias in original studies will be evaluated with the Cochrane revised tool for RCTs (RoB2) and with the risk of bias in non-randomised studies of interventions (ROBINS-I) tool. If for a given practice, more than two original studies on our primary outcome are identified, we will conduct meta-analysis using a random effects model and assess heterogeneity using the $I^{2}$ index. We will assess credibility of evidence (I-IV) based on statistical significance, sample size, heterogeneity and bias as per published criteria.

Ethics and dissemination Ethics approval is not required as original data will not be collected. Knowledge users from three level I trauma centres are involved in the design and conduct of the study in accordance with an integrated knowledge translation approach. Findings related to the rapid review will be available in May 2020. They will be presented to key stakeholders to inform discussions and raise awareness on low-value injury care. In addition, results will be disseminated in a peer-reviewed journal, at national and international scientific meetings and to healthcare associations.

\section{INTRODUCTION}

Orthopaedic injuries affect $89 \%$ of adult trauma patients. ${ }^{1}$ Such injuries are characterised by osseous, soft-tissue, neural or vascular

\section{Strengths and limitations of this study}

- Comprehensive review of evidence on low-value practices in orthopaedic trauma.

- Results will be used to inform discussions and increase awareness of low-value injury care for injury admissions.

- Adopts an integrated knowledge translation approach to ensure uptake of results by trauma stakeholders.

- For some practices, we may identify no evidence or only low-level evidence.

damages to the extremities, pelvis or spine, greatly impacting the person's physical functioning and quality of life, while being associated with a loss in productivity of nearly US $\$ 50$ billion in the USA annually. ${ }^{2}$ Considering the complexity of orthopaedic injuries and the lengthy healing process associated with this type of trauma, referral to specialists such as orthopaedic surgeons as well as multiple radiological imaging and follow-up medical appointments are often recognised as standards of care. Orthopaedic injuries represent the most expensive non-fatal trauma ${ }^{3}$ with total direct and indirect costs of up to US $\$ 456$ billion in the USA annually. ${ }^{4}$

Up to $30 \%$ of healthcare budgets are spent on potentially unnecessary care. ${ }^{5}$ Low-value practices are defined as 'the common use of a particular intervention when the benefits don't justify the potential harm or $\operatorname{cost}^{6}$ and comprise unnecessary hospitalisations, transfers, consultations, diagnostic and therapeutic procedures. ${ }^{5-12}$ Best practice guidelines to optimise outcomes in adult patients with orthopaedic injuries have been published by recognised orthopaedic and trauma organisations (American Academy of Orthopaedic Surgeons, ${ }^{13}$ Orthopaedic Trauma Association, ${ }^{14}$ American College of SurgeonsCommittee on Trauma ${ }^{15}$ and Eastern 
Association for the Surgery of Trauma ${ }^{16}$ ). The emphasis of these guidelines is on the adherence to processes of care (underuse). No evidence-based recommendations are available yet on low-value practices (overuse) in the context of orthopaedic injuries. ${ }^{17}$ Low-value injury care in orthopaedic injuries can lead to inefficient use of specialised resources. More importantly, it can expose patients to unnecessary radiation and to adverse events such as postoperative infection and impaired daily functioning. ${ }^{11}$

A recent scoping review and expert consultation survey ${ }^{17}$ identified 10 potentially low-value intra-hospital practices in the adult orthopaedic trauma population (table 1). A further 23 practices were classified in the grey zone, that is, less than $70 \%$ of experts considered them to be clearly or potentially of low value. After a second round of consultation in which experts were asked which of the 23 grey zone practices they strongly felt should go on to the evidence synthesis phase, five additional practices were retained (table 1). These practices targeted initial and post-treatment diagnostic interventions (ie, imaging and blood tests), initial and follow-up consultation with an orthopaedic surgeon and immobilisation. They represent potential indicators of overuse and could inform the development of de-implementation interventions in the treatment of orthopaedic injuries. However, before recommendations can be made, we need to appraise the available evidence for these practices. The aim of this study is, therefore, to synthesise the evidence on intra-hospital practices for orthopaedic injuries, previously identified as potentially low value, based on predetermined Population-Intervention- Comparator-Outcome-Study design (PICOS) questions (table 1).

\section{METHODS AND ANALYSIS}

We will use a rapid review approach ${ }^{18}$ to synthesise a large body of evidence in 'a timely and credible manner'. Rapid reviews are an assessment of what is known about a practice issue, making concessions to the traditional breadth and depth of the systematic review process. ${ }^{19}$ Hence, to produce timely, high-level evidence, we will limit study designs to the highest available level of evidence and restrict our search to published literature (table 2). All other aspects of the review will follow the Cochrane Handbook for Systematic Reviews of Interventions. ${ }^{20}$ We have reported this protocol using the applicable Preferred Reporting Items for Systematic Reviews and Meta-Analysis Protocols (PRISMA-P) checklist (online supplementary digital file 1). ${ }^{21}$

\section{Eligibility criteria}

Eligibility criteria specific to each practice were developed using the PICOS framework by the project steering committee and expert orthopaedic surgeons (table 1). To ensure the feasibility of reviewing a large body of evidence, we will 'identify studies that will provide the most rigorous evidence to answer the question' as per recommendations on rapid reviews. ${ }^{19}$ Thus, we will use staged study inclusion criteria using published levels of evidence based on the type of study (ie, systematic reviews, randomised controlled trials (RCTs) and then studies with other designs) and study quality (online supplementary digital file 2) ${ }^{22}$ No restriction on date and language will be applied.

\section{Outcomes}

Primary and secondary outcomes were identified for each practice by the project steering committee and clinical experts (table 1). Primary outcomes will include missed injuries (eg, fracture or luxation/subluxation identified on follow-up X-ray or other imaging tests), fracture malunion or non-union, change in clinical management (eg, surgery and follow-up with a specialist) and functional recovery measured with validated tools (eg, Roland Morris Disability Scale and Upper Extremity Functional Index) ${ }^{23-29}$ Secondary outcomes will include pain (eg, Numerical Pain Rating Scale and Brief Pain Inventory) and quality of life measured with validated tools (eg, Numerical Pain Rating Scale, Brief Pain Inventory, 36-item short-form health survey (SF-36) and EuroQol-5 dimension (EQ-5D)) ${ }^{30-34}$ complications (eg, neurological deficits and skin lesions), healthcare service utilisation (eg, reduction in the number of X-rays or other imaging tests, hospitalisations and specialist consultations) and return to work (table 1). Economic evaluations (cost effectiveness) are the subject of another review currently underway. In line with rapid review methodology, outcome definitions may be refined on consultation with the literature. ${ }^{19}$

\section{Search strategy}

On consultation with an information specialist, we will develop search strategies separately for each practice. We will search Medline, EMBASE, the Cochrane Central Register of Controlled Trials and Epistemonikos from their inception up to a maximum of 6 months prior to submission of the article for publication. We will also check reference lists of reviews and retrieved articles for additional studies, and we will perform citation searches on key articles. Since this rapid review aims to synthetise the evidence based on published studies, we would not search the grey literature. Medical subject headings $(\mathrm{MeSH})$ and text word terms will be used and Boolean operators will be applied to combine them. If studies are written in other languages than those spoken by the research team (English, French, German and Arabic) and are judged as relevant based on the abstract, Canadian linguistic healthcare agencies will be consulted for translation (http://www.jhsb.ca/fr/acces-linguistique/ banque-interpretariat). An example of a search strategy for a specific practice is presented in table 2 .

\section{Selection process}

We will manage all citations with EndNote software (V.X8.2, Clarivate Analytics, 2014). We will identify and remove duplicates using electronic and manual screening. To ensure reliability when selecting studies 
Table 1 Population-Intervention-Comparator-Outcome-Study design for the selected low-value practices in orthopaedic injuries

\section{\# Low-value practice (research question)}

Initial diagnostic interventions

$1 \quad$ Population: adults with suspected wrist injury negative on a validated clinical decision rule (eg, Amsterdam Wrist Rule)

Intervention: routine wrist X-ray

Comparator: none or no wrist X-ray

Primary outcome: missed injuries (eg, fracture or luxation/subluxation identified on follow-up X-ray or other imaging tests)

Secondary outcomes: change in clinical management (eg, surgery and follow-up with a specialist) and healthcare resource utilisation (eg, reduction in the number of X-rays or other imaging tests)

Study design: systematic review, RCT, quasi-experimental and observational

$2 \quad$ Population: adults with suspected knee injury negative on a validated clinical decision rule (eg, Ottawa Knee Rule, Pittsburgh)

Intervention: routine knee X-ray

Comparator: none or no knee X-ray

Primary outcome: missed injuries (eg, fracture or luxation/subluxation identified on follow-up X-ray or other imaging tests)

Secondary outcomes: change in clinical management (eg, surgery and needs follow-up with a specialist) and healthcare resource utilisation (eg, reduction in the number of $\mathrm{X}$-rays or other imaging tests)

Study design: systematic review, RCT, quasi-experimental and observational

3 Population: adults with suspected ankle injury negative on a validated clinical decision rule (eg, Ottawa Ankle Rule)

Intervention: routine ankle X-ray

Comparator: none or no ankle X-ray

Primary outcome: missed injuries (eg, fracture or luxation/subluxation identified on follow-up X-ray or other imaging tests)

Secondary outcomes: change in clinical management (eg, surgery and follow-up with a specialist) and healthcare resource utilisation (eg, reduction in the number of X-rays or other imaging tests)

Study design: systematic review, RCT, quasi-experimental and observational

$4 \quad$ Population: adults with blunt single-system trauma and stable with a negative physical exam for pelvic injury Intervention: routine pelvic X-ray

Comparator: none or no pelvic X-ray

Primary outcome: missed injuries (eg, fracture or luxation/subluxation identified on follow-up X-ray or other imaging tests)

Secondary outcomes: change in clinical management (eg, surgery and follow-up with a specialist) and healthcare resource utilisation (eg, reduction in the number of X-rays or other imaging tests)

Study design: systematic review, RCT, quasi-experimental and observational

5 Population: adult trauma, negative on a validated clinical decision rule for spine injury (eg, Canadian C-Spine Rule and National Emergency X-Radiography Utilization Study-NEXUS)

Intervention: routine cervical spine CT or X-ray

Comparator: none or no cervical spine CT or X-ray

Primary outcome: missed injuries (eg, fracture or luxation/subluxation identified on follow-up X-ray or other imaging tests)

Secondary outcomes: change in clinical management (eg, surgery and follow-up with a specialist) and healthcare resource utilisation (eg, reduction in the number of X-rays or other imaging tests)

Study design: systematic review, RCT, quasi-experimental and observational

6 Population: American Society of Anaesthesiologists grade I adults with orthopaedic injury requiring minor surgery

Intervention: preoperative blood tests

Comparator: no preoperative blood tests

Primary outcome: change in clinical management (eg, requests for specialist consultation and follow-up,

medication administration or interruption and administration of blood transfusions)

Secondary outcomes: healthcare resource utilisation (eg, reduction in the number of blood tests)

Study design: systematic review, RCT, quasi-experimental and observational

Initial consultations

Continued 


\begin{tabular}{|c|c|}
\hline$\#$ & Low-value practice (research question) \\
\hline 7 & $\begin{array}{l}\text { Population: adults with isolated (no other T1-L5 injury) thoracolumbar transverse process fracture } \\
\text { Intervention: spine service consultation } \\
\text { Comparator: no spine service consultation } \\
\text { Primary outcome: missed injuries (eg, fracture or luxation/subluxation identified on follow-up X-ray or other } \\
\text { imaging tests) } \\
\text { Secondary outcomes: surgical intervention, functional recovery measured with validated scales (eg, Functional } \\
\text { Independence Measure, Roland Morris Disability Scale and Oswestry Low Back Pain) }{ }^{23-26} \text {, pain measured with } \\
\text { validated scales (eg, Numerical Pain Rating Scale, Brief Pain Inventory and McGill Pain Questionnaire) }{ }^{30-32} \text { and } \\
\text { healthcare resource utilisation (eg, reduction in the number of consultations and hospitalisations) } \\
\text { Study design: systematic review, RCT, quasi-experimental and observational }\end{array}$ \\
\hline
\end{tabular}

Therapeutic interventions

Population: adults with suspected scaphoid fracture with negative CT or MRI*

Intervention: immobilisation (eg, cast, splint, brace and wrap)

Comparator: no immobilisation

Primary outcome: functional recovery (eg, Upper Extremity Functional Index)

Secondary outcomes: pain (eg, Numerical Pain Rating Scale, Brief Pain Inventory and McGill Pain

Questionnaire $)^{30-32}$, return to work, quality of life measured with validated scales (eg, SF-36 and EQ-5D) ${ }^{33} 34$ and healthcare resource utilisation (eg, reduction in the use of healthcare services)

Study design: systematic review, RCT, quasi-experimental and observational

Population: adult fifth metacarpal neck fracture

Intervention: cast immobilisation

Comparator: no cast immobilisation

Primary outcome: functional recovery (eg, Upper Extremity Functional Index ${ }^{27}$

Secondary outcomes: pain (eg, Numerical Pain Rating Scale, Brief Pain Inventory and McGill Pain

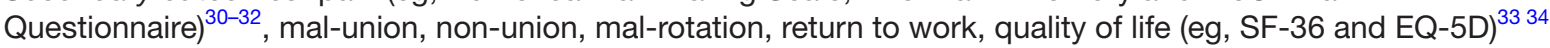
and healthcare resource utilisation (eg, reduction in the use of healthcare services)

Study design: systematic review, RCT, quasi-experimental and observational

Population: A0-A3 thoracolumbar burst fracture and no neurological symptoms

Intervention: orthosis

Comparator: no orthosis

Primary outcome: functional recovery (eg, Functional Independence Measure, Roland Morris Disability Scale and Oswestry Low Back Pain Disability Index) ${ }^{23-26}$

Secondary outcomes: pain (eg, Numerical Pain Rating Scale, Brief Pain Inventory and McGill Pain

Questionnaire) ${ }^{30-32}$, quality of life (eg, SF-36 and EQ-5D) ${ }^{33} 34$, complications (eg, neurological deficits and skin lesions) and healthcare resource utilisation (eg, reduction in the use of healthcare services)

Study design: systematic review, RCT, quasi-experimental and observational

Repeat or post-treatment diagnostic interventions

Population: adults with initial management for isolated closed Mason-Johnson type-I radial head/neck fracture Intervention: routine repeat $\mathrm{X}$-ray

Comparator: none or no repeat X-ray

Primary outcome: fracture mal-union or non-union

Secondary outcomes: change in clinical management (ie, surgery and follow-up with a specialist), functional recovery (eg, Upper Extremity Functional Index and DASH Questionnaire) ${ }^{27} 28$, pain (eg, Numerical Pain Rating Scale, Brief Pain Inventory and McGill Pain Questionnaire) ${ }^{30-32}$ and healthcare resource utilisation (ie, reduction in the number of $\mathrm{X}$-rays or other imaging tests and cost reduction)

Study design: systematic review, RCT, quasi-experimental and observational

Population: adults with fractures treated by operative fixation with a load-sharing construct in good quality bone* $^{*}$

Intervention: routine post-operative X-ray

Comparator: none or no post-operative X-ray

Primary outcome: fracture mal-union

Secondary outcomes: non-union or displacement, change in clinical management (eg, reoperation and increased number of follow-up visits with a specialist), functional recovery (eg, Upper Extremity Functional Index, Lower Extremity Functional Scale and Functional Independence Measure) ${ }^{25} 2729$, pain (eg, Numerical Pain Rating Scale, Brief Pain Inventory and McGill Pain Questionnaire) ${ }^{30-32}$, healthcare resource utilisation (eg, reduction in the number of $\mathrm{X}$-rays or other imaging tests)

Study design: systematic review, RCT, quasi-experimental and observational 


\begin{tabular}{|c|c|}
\hline \# & Low-value practice (research question) \\
\hline 13 & $\begin{array}{l}\text { Population: postsplinting X-ray in adults with non-displaced and minimally displaced fractures with no } \\
\text { manipulation before or during immobilisation* } \\
\text { Intervention: routine post splinting X-ray } \\
\text { Comparator: none or no post-splinting X-ray } \\
\text { Primary outcome: fracture mal-union, non-union or displacement } \\
\text { Secondary outcomes: change in clinical management (eg, surgery and increased number of follow-up visits } \\
\text { with a specialist), functional recovery (eg, Upper Extremity Functional Index, DASH Questionnaire and Lower } \\
\text { Extremity Functional Scale) }{ }^{27}{ }^{28} \text {, pain (eg, Numerical Pain Rating Scale, Brief Pain Inventory and McGill Pain } \\
\text { Questionnaire) }{ }^{30-32} \text { and healthcare resource utilisation (eg, reduction in the number of X-rays or other imaging } \\
\text { tests). } \\
\text { Study design: systematic review, RCT, quasi-experimental and observational }\end{array}$ \\
\hline
\end{tabular}

\section{Follow-up consultations}

Population: adults with adequately aligned fifth metacarpal fracture*
Intervention: routine follow-up consultation
Comparator: none or no follow-up consultation
Primary outcome: functional recovery (eg, Upper Extremity Functional Index and Functional Independence
Measure) ${ }^{25} 27$
Secondary outcomes: fracture mal-union, non-union or displacement, pain (eg, Numerical Pain Rating Scale,
Brief Pain Inventory and McGill Pain Questionnaire), ${ }^{30-32}$ quality of life (eg, SF-36 and EQ-5D) ${ }^{33} 34$ and healthcare
resource utilisation (eg, reduction in the use of healthcare services)
Study design: systematic review, RCT, quasi-experimental and observational
Population: adults with fifth metatarsal fracture managed non-operatively
Intervention: routine follow-up consultations
Comparator: none or no follow-up consultation
Primary outcome: functional recovery (eg, Upper Extremity Functional Index and Functional Independence
Measure) ${ }^{25-27}$
Secondary outcomes: fracture mal-union, non-union or displacement, pain (eg, Numerical Pain Rating Scale,
Brief Pain Inventory and McGill Pain Questionnaire) ${ }^{30-32}$, quality of life (eg, SF-36 and EQ-5D) ${ }^{33} 34$ and healthcare
resource utilisation (eg, reduction in the use of healthcare services)
Study design: systematic review, RCT, quasi-experimental and observational

*Practices added to the evidence synthesis following a second round of consultation with expert clinicians.

DASH, Disabilities of the Arm, Shoulder and Hand; EQ-5D, EuroQol-5 dimension; RCT, randomised controlled trial; SF-36, 36-item short-form health survey.

for a given practice, all reviewers will assess a series of 100 titles in rounds until acceptable agreement has been achieved. ${ }^{20}$ Pair of reviewers (MB and LM, and CC and PAT) will then independently screen all identified records using titles, abstracts and full texts using Covidence software. Any disagreement will be resolved through discussion between reviewers and a senior author (JP) will be consulted if necessary. Included and excluded studies will be described in a PRISMA flow chart. ${ }^{35}$

\section{Data extraction and management}

Two pairs of experienced reviewers (MB and LM, and CC and PAT) will work independently to extract data using a standardised data extraction form piloted on five studies by each reviewer as recommended by the

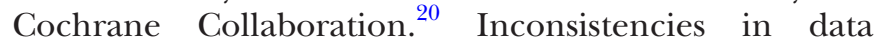
extraction will be resolved by discussion between the two reviewers, and, if consensus is not reached, with a senior author (JP). Extracted data will include information on study design, setting and year, characteristics of the study population (age and sex, injury mechanism, severity and type of injury, and associated injuries), intervention(s), comparator(s), outcome(s) and measures of effect. When information is available in figures only, we will abstract graphical data using computer-assisted software (Plot Digitizer). ${ }^{36}$ Furthermore, we will contact study authors with up to three email attempts when information is unclear or unavailable.

\section{Quality assessment}

Pairs of reviewers will independently critically appraise methodological quality for systematic reviews using the Measurement Tool to Assess Systematic Reviews version 2 (AMSTAR-2) ${ }^{37}$ and the risk of bias for original studies using the Cochrane revised tool for RCTs (RoB2) ${ }^{38}$ and the risk of bias in non-randomised studies of interventions (ROBINS-I) ${ }^{39}$ tool for observational studies. Risk of bias will be categorised as low, moderate, high and unclear.

\section{Synthesis}

For each practice, we will report the number of studies according to their design, sample sizes, methodological quality (systematic reviews) or risk of bias (original studies), and effect estimates for primary and secondary 


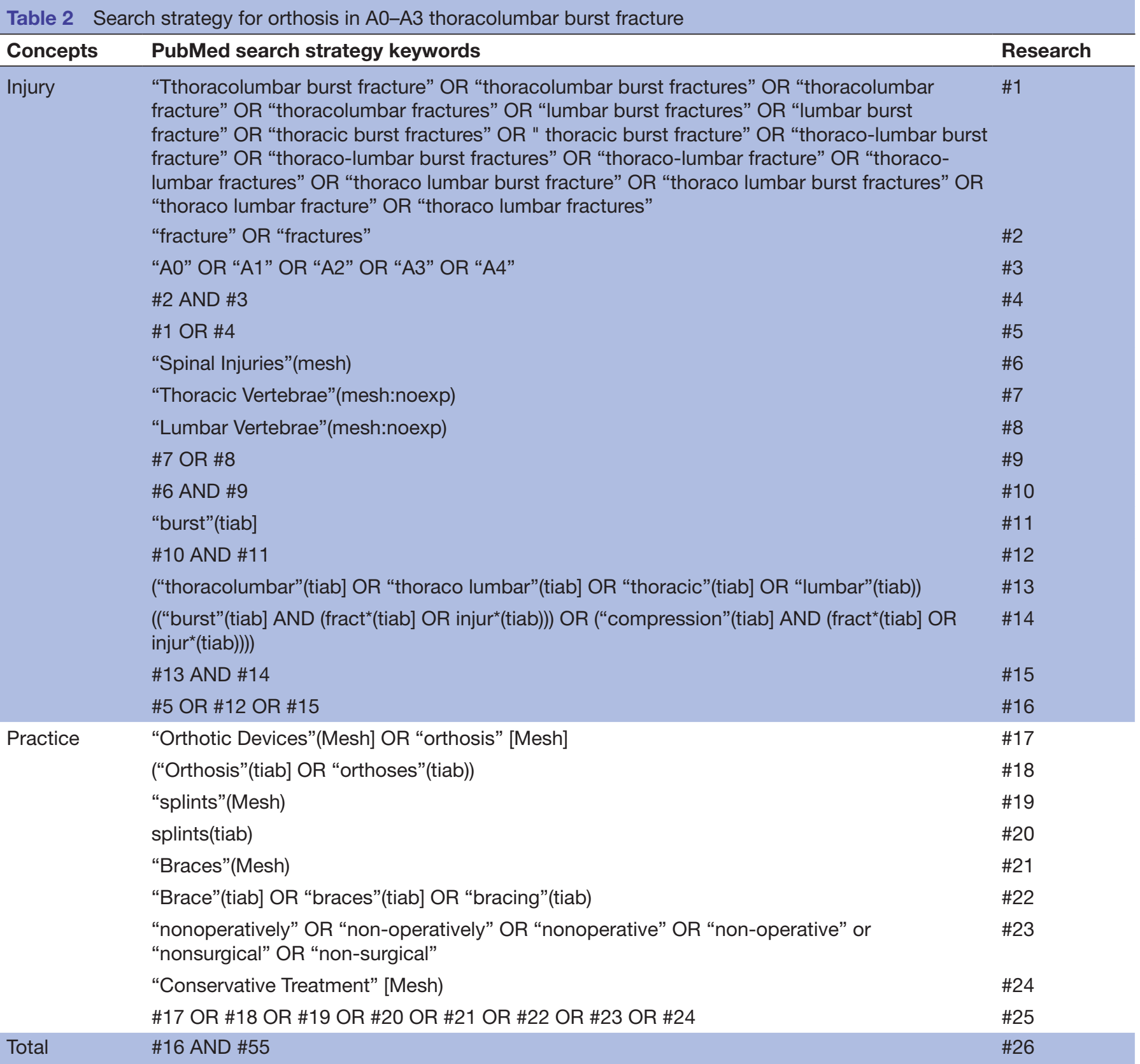

outcomes. We will use a tabular and graphical (forest plot) approach to facilitate knowledge transfer to stakeholders.

\section{Statistical analyses}

If, for a given practice, more than two original studies with the same outcome are identified, we will conduct meta-analysis using random effects models. We will use Review Manager ${ }^{40}$ to calculate pooled weighted mean differences (continuous data) or risk ratios (binary data) and $95 \%$ CIs. If the number of studies is sufficient, we will conduct sensitivity analyses restricted to studies at low risk of bias for primary outcomes. We will assess heterogeneity using the $\mathrm{I}^{2}$ index.

\section{Credibility of evidence}

For practices with published meta-analyses or original studies allowing meta-analyses, we will evaluate credibility of evidence using previously published criteria. ${ }^{41}{ }^{42}$ These criteria class evidence from meta-analyses into five categories: convincing (class I), highly suggestive (class II), suggestive (class III), weak (class IV) and non-significant (NS), based on the statistical significance of pooled and original study estimates, sample size, heterogeneity and bias. For example, to be judged convincing, the evidence must exist from both observational studies and RCTs, and measures of association should be of same direction, statistically significant at $p \leq 0.001$ and free from bias. While to be judged NS, evidence from observational studies or RCTs is enough to conclude that a substantial effect is unlikely based on the magnitude and the significance level. ${ }^{41}$

\section{Potential limitations}

This rapid review has some limitations. Although we aim to select studies that use the most robust methodology 
to evaluate the effect of low-value practices, for some practices we may identify no evidence or only low-level evidence. Nonetheless, research gaps could be identified by documenting the type of study designs used and methodological quality/risk of bias. Given our rapid review design, we will not search the grey literature. However, we do not anticipate that this will lead us to miss any highlevel evidence.

\section{Potential impact}

This rapid review is the first to evaluate the evidence on low-value care in orthopaedic injuries. Our evidence synthesis will be presented to key stakeholders to inform discussions and increase awareness on low-value care. Considering the high volume of patients who sustain orthopaedic injuries annually, the reduction of low-value practices in this population has the potential to free up an important quantity of resources and more importantly, to optimise outcomes in patients who need to receive specialised treatments. Healthcare services quality and efficiency could be greatly improved.

\section{ETHICS AND DISSEMINATION}

Ethics approval is not required as original data will not be collected. Findings related to the rapid review should be available in May 2020 ( 7 months from study onset in September 2019). They will be disseminated in a peerreviewed journal, at national and international scientific meetings and to clinical and policy stakeholders (Choosing Wisely Canada, Trauma Association of Canada, Orthopaedic Trauma Association, American College of Surgeons-Committee on Trauma, Institut national d'excellence en santé et en services sociaux).

\section{Patient and public involvement}

No patient or public representatives will be involved in this study.

\section{Author affiliations}

${ }^{1}$ Population Health and Optimal Health Practices Research Unit, Trauma Emergency - Critical Care Medicine, Centre de Recherche du CHU de Québec Université Laval (Hôpital de l'Enfant-Jésus), Québec City, Québec, Canada

${ }^{2}$ Faculty of Nursing, Université Laval, Québec City, Québec, Canada

${ }^{3}$ Department of Social and Preventive Medicine, Université Laval, Québec City, Québec, Canada

${ }^{4}$ Division of Orthopaedic Surgery, CIUSSS du Nord-de-l'île-de-Montréal (Hôpital du Sacré-Coeur de Montréal), Montréal, Québec, Canada

${ }^{5}$ Division of Orthopaedic Surgery, CHU de Québec-Université Laval, Québec City, Québec, Canada

${ }^{6}$ Division of Orthopaedic Surgery, McGill University Health Center, Montréal, Québec, Canada

${ }^{7}$ Division of Neurosurgery, CHU de Québec - Université Laval, Québec City, Québec, Canada

\section{Twitter Mélanie Bérubé @BrubMlanie1 and Lynne Moore @Moore}

Contributors MB contributed to the elaboration of keywords and the search strategy, drafted the manuscript and approved the final version of the manuscript. LM led the development of the protocol and drafted the manuscript with the first author. She acts as guarantor for the rapid review. SL contributed to the development of PICOS questions, revised the manuscript and approved the final version. IF contributed to the elaboration of keywords, the search strategy and the data extraction form, revised the manuscript and approved the final version. ML and JP contributed to the identification of low-value injury care, development of PICOS questions, critically revised the manuscript and approved the manuscript. JL contributed to the identification of low-value injury care, revised the manuscript and approved the final version. SP and GB contributed to the identification of low-value injury care, critically revised the manuscript and approved the final version. PAT and CC contributed to the data extraction form development, critically revised the manuscript and approved the manuscript.

Funding This research was supported by the Canadian Institutes of Health Research (Foundation grant, \#353374).

Competing interests None declared.

Patient consent for publication Not required.

Provenance and peer review Not commissioned; externally peer reviewed.

Open access This is an open access article distributed in accordance with the Creative Commons Attribution Non Commercial (CC BY-NC 4.0) license, which permits others to distribute, remix, adapt, build upon this work non-commercially, and license their derivative works on different terms, provided the original work is properly cited, appropriate credit is given, any changes made indicated, and the use is non-commercial. See: http://creativecommons.org/licenses/by-nc/4.0/.

\section{ORCID iDs}

Mélanie Bérubé http://orcid.org/0000-0002-6657-3915

Pier-Alexandre Tardif http://orcid.org/0000-0003-3003-2399

\section{REFERENCES}

1 American College of Surgeons Committee on Trauma Leadership. National trauma Databank 2016 annual report, 2016. Available: https://www.facs.org/ /media/files/quality\%20programs/trauma/ ntdb/ntdb\%20annual\%20report\%202016.ashx

2 Center for Disease Control and Prevention - National Center for Injury Prevention and Control. Data \& Statistics - Cost of Injury Reports (Nonfatal Emergency Department Treated and Released Injuries and Nonfatal Hospitalized Injuries - Extremity and Neck Injuries), 2010. Available: https://wisqars.cdc.gov:8443/costT/

3 Miller TR, Lestina DC. Patterns in US medical expenditures and utilization for injury, 1987. Am J Public Health 1996;86:89-93.

4 Center for Disease Control and Prevention. National center for injury prevention and control. Cost of injury care 2017.

5 Reilly BM, Evans AT. Much ado about (doing) nothing. Ann Intern Med 2009;150:270-1.

6 Choosing Wisely Canada, 2015. Available: https:// choosingwiselycanada.org/

7 Boat TF, Chao SM, O'Neill PH. From waste to value in health care. JAMA 2008;299:568-71.

8 Berwick DM, Hackbarth AD. Eliminating waste in US health care. JAMA 2012;307:1513-6.

9 Morgan DJ, Dhruva SS, Coon ER, et al. 2017 update on medical overuse: a systematic review. JAMA Intern Med 2018;178:110-5.

10 Berwick DM. Avoiding overuse-the next quality frontier. Lancet 2017;390:102-4.

11 Brownlee S, Chalkidou K, Doust J, et al. Evidence for overuse of medical services around the world. Lancet 2017;390:156-68.

12 Saini V, Brownlee S, Elshaug AG, et al. Addressing overuse and underuse around the world. Lancet 2017;390:105-7.

13 American Academy of Orthopaedic Surgeons. Ortho guidelines. Available: http://www.orthoguidelines.org/guidelines [Accessed June $12019]$.

14 Orthopaedic Trauma Association. Evidence-Based medicine resource list. Available: https://ota.org/education/evidence-basedmedicine-resource-list [Accessed June 1 2019].

15 American College of Surgeons - Trauma Quality Improvement Program. Best practices guidelines in the management of orthopaedic trauma. Available: https://www.facs.org/ /media/files/ quality\%20programs/trauma/tqip/tqip $\% 20 \mathrm{bpgs} \% 20$ in $\% 20$ the $\%$ 20management\%20of\%20orthopaedic\%20traumafinal.ashx [Accessed June 12019$]$

16 Eastern Association for the Surgery of Trauma. East practice management guidelines. Available: https://www.east.org/education/ practice-management-guidelines [Accessed June 1 2019].

17 Moore L, Lauzier F, Tardif P-A, et al. Low-Value clinical practices in injury care: a scoping review and expert consultation survey. $J$ Trauma Acute Care Surg 2019;86:983-99.

18 Tricco AC, Antony J, Zarin W, et al. A scoping review of rapid review methods. BMC Med 2015;13:224. 
19 Tricco AC, Langlois EV, Straus SE, eds. Rapid Reviews to Strengthen Health Policy and Systems: A Practical Guide. Geneva: World Health Organization, 2017License: CC BY-NC-SA 3.0 IGO.

20 Higgins J, Thomas S, eds. Cochrane Handbook for Systematic Reviews of Interventions Version 6. The Cochrane Collaboration, 2019. https://training.cochrane.org/handbook/current

21 Moher D, Shamseer L, Clarke M, et al. Preferred reporting items for systematic review and meta-analysis protocols (PRISMA-P) 2015 statement. Syst Rev 2015;4:1.

22 Slobogean G, Bhandari M, Mohit B. Introducing levels of evidence to the Journal of orthopaedic trauma: implementation and future directions. J Orthop Trauma 2012;26:127-8.

23 Roland M, Morris R. A study of the natural history of back pain. Part I: development of a reliable and sensitive measure of disability in lowback pain. Spine 1983;8:141-4.

24 Davies CC, Nitz AJ. Psychometric properties of the Roland-Morris disability questionnaire compared to the Oswestry disability index: a systematic review. Physical Therapy Reviews 2009;14:399-408.

25 Linacre JM, Heinemann AW, Wright BD, et al. The structure and stability of the functional independence measure. Arch Phys Med Rehabil 1994;75:127-32.

26 Fairbank JC, Pynsent PB. The Oswestry disability index. Spine 2000;25:2940-53.

27 Gabel CP, Michener LA, Burkett B, et al. The upper limb functional index: development and determination of reliability, validity, and responsiveness. J Hand Ther 2006;19:328-49.

28 Beaton DE, Katz JN, Fossel $\mathrm{AH}$, et al. Measuring the whole or the parts? validity, reliability, and responsiveness of the disabilities of the arm, shoulder and hand outcome measure in different regions of the upper extremity. J Hand Ther 2001;14:128-46.

29 Binkley JM, Stratford PW, Lott SA, et al. The lower extremity functional scale (LEFS): scale development, measurement properties, and clinical application. North American orthopaedic rehabilitation research network. Phys Ther 1999;79:371-83.

30 Farrar JT, Young JP, LaMoreaux L, et al. Clinical importance of changes in chronic pain intensity measured on an 11-point numerical pain rating scale. Pain 2001;94:149-58.
31 Cleeland CS. The brief pain inventory user guide. The University of Texas, M.D. Anderson Cancer Center, 2009.

32 Melzack R. The McGill pain questionnaire: major properties and scoring methods. Pain 1975;1:277-99.

33 Ware JE, Sherbourne CD. The mos 36-item short-form health survey (SF-36). I. conceptual framework and item selection. Med Care 1992;30:473-83.

34 Dolan P. Modeling valuations for EuroQol health states. Med Care 1997;35:1095-108.

35 Cochrane Consumers and Communication Review Group. Data extraction template for included studies version 1.7, 2016. Available: http://cccrg.cochrane.org/ author-resources

36 Jelicic Kadic A, Vucic K, Dosenovic S, et al. Extracting data from figures with software was faster, with higher interrater reliability than manual extraction. J Clin Epidemiol 2016;74:119-23.

37 Shea BJ, Reeves BC, Wells G, et al. AMSTAR 2: a critical appraisal tool for systematic reviews that include randomised or nonrandomised studies of healthcare interventions, or both. BMJ 2017;358:j4008.

38 Higgins JPT, Sterne JAC, Savović J. A revised tool for assessing risk of bias in randomized trials. In: Chandler J, McKenzie J, Boutron I, eds. Cochrane methods. Cochrane database Syst Rev. 10:(Supp1), 2016.

39 Sterne JA, Hernán MA, Reeves BC, et al. ROBINS-I: a tool for assessing risk of bias in non-randomised studies of interventions. BMJ 2016;355:i4919.

40 Review Manager (RevMan). Computer program. version 5.3. Copenhagen: The Nordic Cochrane Centre, The Cochrane Collaboration, 2014

41 Theodoratou E, Tzoulaki I, Zgaga L, et al. Vitamin D and multiple health outcomes: umbrella review of systematic reviews and meta-analyses of observational studies and randomised trials. BMJ 2014;348:g2035.

42 Li X, Meng X, Timofeeva M, et al. Serum uric acid levels and multiple health outcomes: umbrella review of evidence from observational studies, randomised controlled trials, and Mendelian randomisation studies. BMJ 2017;357:j2376. 